\title{
Recurrence of DiGeorge syndrome: prenatal detection by FISH of a molecular 22q11 deletion
}

\author{
J O Van Hemel, C Schaap, D Van Opstal, M P Mulder, M F Niermeijer, \\ J H C Meijers
}

\begin{abstract}
We report on a prenatal diagnosis by FISH of a familial 22q11 deletion associated with DiGeorge syndrome (DGS). The deletion was seen in the proband with symptoms of full DGS, in the physically normal father, and in a subsequent pregnancy. After birth this child showed hypocalcaemia, a $T$ cell deficit, and a right sided aortic arch.
\end{abstract}

( $($ Med Genet 1995;32:657-658)

The DGS involves conotruncal heart defects, hypoplastic or absent thymus and parathyroids, and facial dysmorphism (McKusick No $188400)$. A deletion of a gene or a group of contiguous genes from $22 \mathrm{q} 11$ is probably the cause of this disorder. This is supported by the detection in DGS children of unbalanced chromosomal translocations ${ }^{1}$ or microdeletions, ${ }^{2}$ mostly involving $22 \mathrm{q} 11$. Deletions of specific DNA sequences have also been described. ${ }^{34}$ Approximately $8 \%$ of index patients with DGS show familial transmission of the $22 \mathrm{q} 11$ deletion. ${ }^{4}$ Noticeably, most of the parents with deletions have only minor symptoms or only signs of learning disability or mild mental retardation. Prenatal diagnosis applying fluorescence in situ hybridisation (FISH) with 22q11 specific probes has been performed, ${ }^{4}$ with normal outcome.

We offered genetic counselling to a couple whose second child had died two weeks after birth. This girl had the typical symptoms of complete DGS. She had a truncus arteriosus communis type II with atrioventricular septal defect and a right sided aortic arch. Other symptoms were neonatal hypocalcaemia and $\mathrm{T}$ cell disturbances. At necropsy only one parathyroid gland and a small hypoplastic thymus were present. High resolution cytogenetic studies performed on her fibroblasts (cell line F9231 ; 93RD59) showed a deletion at 22q11, which was confirmed by applying FISH with probe M51 that detects a molecular deletion in the DGS critical region of chromosome $22^{5}$ (figure). Both parents were physically normal, although they both had mild learning disabilities, especially the father. In childhood he had recurrent upper airway infections and was frequently admitted to hospital. However, no data could be obtained about possible im- munological disturbances. The father had a tendency to depression and alcohol abuse. No microdeletions were visible in lymphocyte metaphases of either parent. After the mother became pregnant, she asked for prenatal diagnosis. FISH on lymphocyte metaphases from both parents and fetal amniotic cells was done with M51. The molecular deletion was detected in the male fetus and in its father. These deletions were not visible cytogenetically.

The parents were informed about the risk for DGS, velocardiofacial syndrome (VCFS), cardiac defects, and mental retardation. ${ }^{67}$ The parents decided to continue the pregnancy. Ultrasound studies in the 20th and 23rd week of pregnancy did not show cardiac or other anomalies. The boy was born at term and developed hypocalcaemia with low parathyroid hormone levels. $T$ cell function studies indicated a moderate $\mathrm{T}$ cell deficit. Echocardiography showed a right sided aortic arch without intracardiac anomalies. Now, at 6 months of age, his clinical condition is stable.

This is the first reported prenatal diagnosis of a molecular deletion in the DGS critical region of chromosome 22 . Such a deletion is associated with a spectrum of malformations covered by the acronym CATCH $22 .^{8}$ The anomalies seen in this family show the phenotypic variability of the M51 deletion. The anomalies of the youngest boy are less severe than those of the proband, with psychiatric problems in the father. A variety of psychiatric problems have been described in VCFS patients. ${ }^{9}$ After detection of the $22 \mathrm{q}$ deletion in the father additional investigations were performed. Serum calcium and immunological screening, including IgG, IgA, IgM, IgG subclasses, and complement reactions ( $\mathrm{CH} 50)$ were all normal. Intracardiac abnormalities were excluded by echocardiography; the only abnormality was a right sided descending aorta detected by $x$ ray of the chest.

In this family and others, the range of phenotypes associated with the molecular deletions detected with probe M51 complicates genetic counselling. The classical DGS syndrome is usually sporadic but may be transmitted as an autosomal dominant trait; Shprintzen syndrome is usually autosomal dominant. We propose that parents with a proven molecular deletion are counselled as having an increased risk for cardiac defects, DGS, immunological disturbance, and cleft palate, occurring as single abnormalities or in syndromic forms. \\ Revised ver 7}




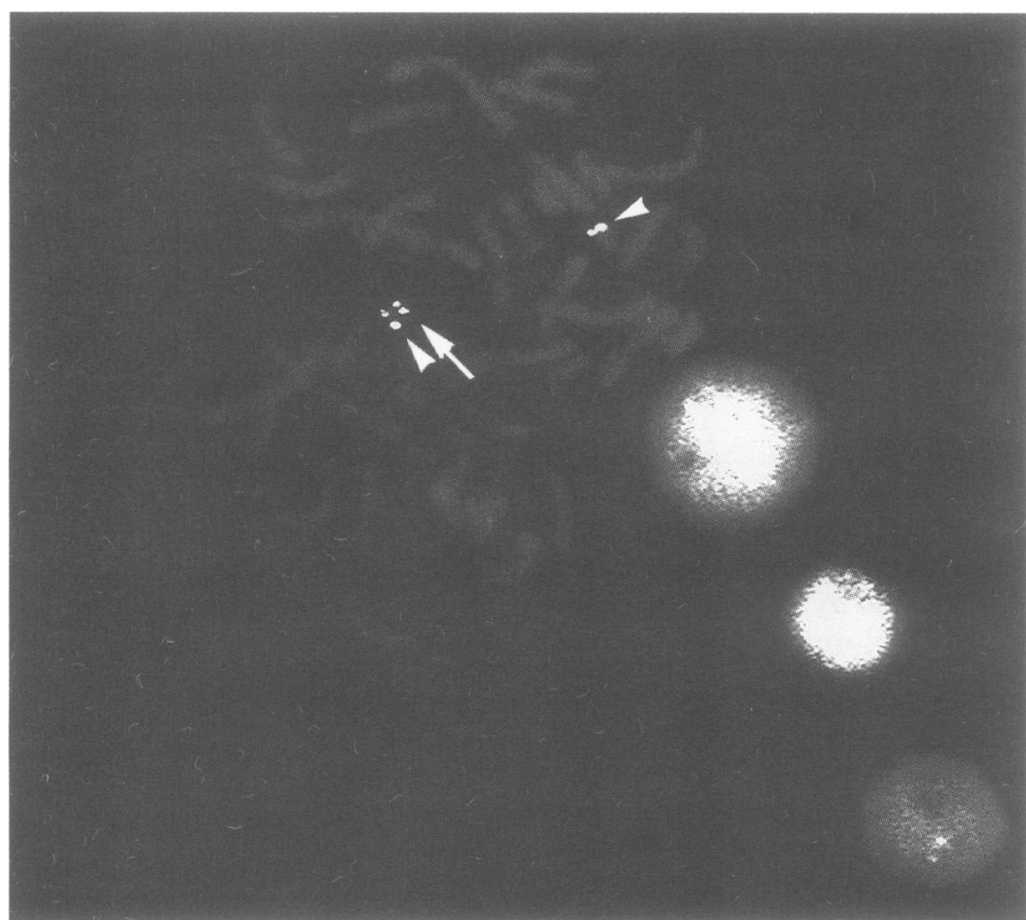

Part of a metaphase after FISH with cosmid probe M51, region $22 q 11$ (arrow) and cosmid probe M69, region 22q13.3 (arrowheads), the latter used for recognition of chromosome 22. Only one chromosome 22 shows the M51 signals, pointing to a deletion in the homologous 22 .
For the prenatal diagnosis of microdeletion associated syndromes like DGS, FISH appears to be a rapid and reliable method. ${ }^{10}$ It may also be reliably applied in chorionic villus mitoses which usually give a lesser quality of their chromosomes. An early first trimester diagnosis is important as an option during genetic counselling when there is a $50 \%$ recurrence risk of familial DGS, ${ }^{7}$ after careful explanation to the parents of the substantial variability of this disorder.

We thank Bert Eussen and Annet van der Heiden for skilful laboratory work, and Professors H Galjaard and J C Molenaar for their continuous support.

1 Greenberg F. DiGeorge syndrome: an historical review of linical and cytogenetic features. $\mathcal{F}$ Med Genet 1993;30: 803-6.

2 Wilson DI, Cross IE, Goodship JA, et al. A prospective cytogenetic study of 36 cases of DiGeorge syndrome. $\mathrm{Am}$ f Hum Genet 1992;51:957-63.

3 Carey AH, Kelly D, Halford S, et al. Molecular genetic study of the frequency of monosomy 22q11 in DiGeorge syndrome. Am $\ngtr$ Hum Genet 1992;51:964-70.

4 Driscoll DA, Salvin J, Sellinger B, et al. Prevalence of 22q11 microdeletions in DiGeorge and velocardiofacial 2q11 microdeletions in DiGeorge and velocardiofacial syndromes: implications for genetic counselling and pre-
natal diagnosis. $f$ Med Genet 1993;30:813-17.

5 Mulder MP, Wilke M, Langeveld M, et al. Positional mapping of loci in the DiGeorge critical region at chromosome 22 11 using a new marker (D22S183). Hum Genet (in press).

6 Driscoll DA, Budarf ML, Emanuel BS. Antenatal diagnosis of DiGeorge syndrome. Lancet 1991;338:1390.

7 Wilson DI, Goodship JA, Burn J, Cross IE, Scambler PJ. Deletions within chromosome 22q11 in familial congenital heart disease. Lancet 1992;340:573-5.

8 Wilson DI, Burn J, Scambler P, Goodship J. Syndrome of the month. DiGeorge syndrome, part of CATCH $22 . \mathcal{J}$ Med Genet 1993;30:852-6.

Further studies are needed to determine the potential of probe M51 to differentiate between isolated and familial forms of congenital heart defects with a very low or a $50 \%$ recurrence risk, respectively. 